\title{
Dark energy from quantum uncertainty of distant clock
}

\section{M.J. Luo}

\author{
Department of Physics, Jiangsu University, \\ Zhenjiang 212013, People's Republic of China \\ E-mail: mjluo@ujs.edu.cn
}

ABSTRACT: The observed cosmic acceleration was attributed to an exotic dark energy in the framework of classical general relativity. The dark energy behaves very similar with vacuum energy in quantum mechanics. However, once the quantum effects are seriously taken into account, it predicts a completely wrong result and leads to a severe fine-tuning. To solve the problem, the exact meaning of time in quantum mechanics is reexamined. We abandon the standard interpretation of time in quantum mechanics that time is just a global parameter, replace it by a quantum dynamical variable playing the role of physical clock. We find that synchronization of two spatially separated clocks can not be precisely realized at quantum level. There is an intrinsic quantum uncertainty of distant clock time, which implies an apparent vacuum energy fluctuation and gives an observed dark energy density $\rho_{d e}=\frac{6}{\pi} L_{P}^{-2} L_{H}^{-2}$ at tree level approximation, where $L_{P}$ and $L_{H}$ are the Planck and Hubble scale cutoffs. The fraction of the dark energy is given by $\Omega_{d e}=\frac{2}{\pi}$, which does not evolve with the internal clock time. The "dark energy" as a quantum cosmic variance is always seen comparable with the matter energy density by an observer using the internal clock time. The corrected distance-redshift relation of cosmic observations due to the distant clock effect are also discussed, which again gives a redshift independent fraction $\Omega_{d e}=\frac{2}{\pi}$. The theory is consistent with current cosmic observations.

KEYwords: Cosmology of Theories beyond the SM, Models of Quantum Gravity

ARXIV EPRINT: 1401.2488 


\section{Contents}

1 Introduction $\quad 1$

2 Quantum uncertainty of distant clock 2

3 Dynamical system under physical clock 4

3.1 Zero-point energy 5

3.2 Vacuum energy fluctuations 5

$\begin{array}{ll}3.3 & \text { The coincidence problem }\end{array}$

4 Distance-redshift relation $\quad 8$

5 Conclusions $\quad 9$

\section{Introduction}

The most important observational discovery of physics in the past decade is the acceleration of the expanding universe $[1,2]$. In the standard model of cosmology based on the classical general relativity, the mysterious driving force of the acceleration could be simply attributed to a kind of energy unseen before, called dark energy [3]. The observational studies of the dark energy shows that it is (i) almost uniformly distributed, (ii) very slowly varied with time and (iii) the equation of state is around $w=-1$.

If we only consider these three properties of the dark energy, it behaves very similar with the vacuum energy we have already known in quantum mechanics. However, if the quantum nature of vacuum is seriously taken into account, it gives a disappointing wrong prediction to its value [4]. The quantum mechanics predicts that it is quartic divergent up to the ultraviolet cut-off. If the validity of quantum mechanics is believed up to the Planck scale $10^{19} \mathrm{GeV}$, the theory gives a very large prediction $\left(10^{19} \mathrm{GeV}\right)^{4}$, which is about $10^{120}$ times departure to the current observational value $\rho_{d e} \sim\left(10^{-11} \mathrm{GeV}\right)^{4}$.

Compared with the small bare value, the large result would need to be cancelled almost, but not exactly. It seems almost impossible to explain the observed dark energy within the framework of conventional quantum mechanics unless the theory is severely fine-tuned. The shortcoming of the vacuum energy explanation gives room to other attempts to resolve the problem, such as many phenomenological scalar fields dark energy models [5], but unfortunately they are also restricted in classical or semi-classical framework. These kinds of models can also reproduce the above three properties and a correct energy density within current range of observations, by carefully tuning its kinetic term and classical potential to a specific shape. In fact, even any behavior e.g. the time evolution of dark energy and the equation of state around $w=-1$ can be engineered. Actually, without quantum mechanics, a very small cosmological constant, phenomenologically, also poses no problem. So the 
real question of dark energy in fact concerns the inconsistent predictions between quantum mechanics and general relativity.

The dark energy problem is a crisis deeply rooted in the foundation of physics. It is known that the vacuum energy corrections to the particle mass does gravitate [6], and hence there is by now no experimental evidence showing any violation of the equivalence principle. If we trust the equivalence principle, all energies gravitate, why we do not feel the large amount of quantum vacuum energies by their gravitational effect, that is the first part of the problem. It is an obvious contradiction between quantum mechanics and the equivalence principle. If any mechanisms prohibit their gravitational effects, why it seems that the quantum vacuum leaves a small remnant gravitational effect which drives the cosmic acceleration, which is the second part of the problem. Current observations bring forward the third part of the problem: if the dark energy is a constant vacuum energy, it is comparable with the matter energy density only in a particular epoch, since the matter energy density is diluted as the universe expanding, why the current observed vacuum energy is comparable to the matter energy density or critical energy density now, which is known as the coincidence problem or "why now" problem [7].

It would be a "mission impossible" to solve these three aspects of the problem, if our arguments are built upon the two foundations mentioned: (i) standard quantum mechanics and (ii) the equivalence principle of the general relativity. Remind that these two basis by now still have not reconciled with each other, preventing a consistent theory of quantum gravity, so it becomes more or less understandable that these two theories would not give a consistent prediction to the observed cosmic dark energy. The observed dark energy is likely an experimental evidence for the confliction between these two theories. As a general believe, the difficulty of reconciling the quantum mechanics and the general relativity is deeply rooted in the very different treatment of the concept of time [8].

\section{Quantum uncertainty of distant clock}

In the quantum mechanics, time as a global parameter is independent with where the clocks are placed on a space-like hypersurface. But this statement is not true in all rigor when the quantum nature of clocks is taken into account. In the spirit of relativity, time must be operationally defined by a physical clock field $T(x)$ describing the readings of e.g. a pointer's position of the clock, where $x=\left(x_{0}, x_{1}, x_{2}, x_{3}\right) \in \mathbb{R}_{4}$ are external space-time point parameter of the clock field in Euclidean metric. The clock reading $T(x)$ is an internal time measured by a local observer, while the external parameter $x$ can only be measured by an external classical observer outside the universe. The physical clock $T(x)$ is assumed to be a real scalar field, and satisfies a zero-mass free field action,

$$
S_{T}=\int d^{4} x \frac{1}{2}\left(\partial_{x} T\right)^{2} .
$$

Now considering a thought experiment comparing the quantum states of two spatially separated quantum clocks. The two quantum states of the clocks placed at $x$ and $y$ here are described by states $|T(x)\rangle$ and $|T(y)\rangle$. If the norm of the inner product of these two 
quantum states equal to 1 , then these two states are identical, says, these two quantum clocks are completely synchronized. The inner product is easy to calculate according to the clock's action eq. (2.1), when the space-like interval $|x-y|$ is considerable, we find the asymptotic correlation between the clocks

$$
\langle T(x) \mid T(y)\rangle \sim \frac{1}{4 \pi^{2}|x-y|^{2}},
$$

which decays with the distance between two clocks. For there is no prior reason to tell us that whether the "same" clocks spatially separated are precisely synchronized, the gradual decorrelation exhibits that the synchronization between two quantum clocks can not be precisely realized. If we consider the clock at $y$ is standard (zero-uncertainty), then the same clock at $x$ is uncertain. In a homogeneous, isotropic, flat and empty space, considering a standard clock with reading $T(y)$ is transported from place $y$ to $x$, then the wavefunction that one finds the clock at the distant place $x$ with reading $T(x)$ is given by

$$
\int_{T(y)}^{T(x)} \mathcal{D} T e^{-S_{T}}=\frac{V_{\mathbb{R}_{3}}^{2}}{4 \pi^{2}|x-y|^{2}} e^{-2 V_{\mathbb{R}_{3}} \frac{[T(x)-T(y)]^{2}}{|x-y|}}=\frac{1}{\sigma^{4}(2 \pi)^{2}} e^{-\frac{4[T(x)-T(y)]^{2}}{2 \sigma^{2}}}
$$

where $\int \mathcal{D} T$ is the Feynman's path integral of the physical clock. The width $\sigma^{2}$ of the wavefunction describes the uncertainty of the reading $T(x)$ of the distant clock at $x$ with respect to the standard clock at $y$, which is given by

$$
\sigma^{2}=\left\langle\delta T^{2}\right\rangle=\frac{1}{V_{\mathbb{R}_{3}}}|x-y|,
$$

where $V_{\mathbb{R}_{3}}$ is the 3 -volume infrared cut-off. Therefore, the simultaneity defined by physical clock $\langle T\rangle=$ constant has an intrinsic quantum uncertainty increasing with the spatial interval $\left\langle\delta T^{2}\right\rangle \propto|x-y|$. Since the infrared cut-off 3-volume $V_{\mathbb{R}_{3}}$ here is considered to be the cosmic scale but not infinity, the uncertainty of simultaneity is not zero. It is a so small number that it can be ignored in our ordinary observation, while it is considerable and important when the spatial interval is at cosmic scale. By dimensional consideration, the distant simultaneity uncertainty can be written as

$$
\left\langle\delta t^{2}\right\rangle \sim L_{H}^{-3} L_{P}^{4}|x-y|
$$

where $L_{H} \sim V_{\mathbb{R}_{3}}^{1 / 3}$ and $L_{P}$ are the infrared and ultraviolet cut-offs chosen as the Hubble and Planck scale. The formula provides a universal limit to distant time measurement. In general, if we consider the time is measured by a quantum physical clock, but a global parameter, an intrinsic quantum uncertainty of distant simultaneity is inevitable. It is worth emphasizing: (i) the effect is different from the time dilation, it does not change the central value $\langle t\rangle$ of the distant time, it only makes the time fuzzy with a non-vanishing $\left\langle\delta t^{2}\right\rangle$. (ii) Different from those time effects predicted from relativity, in which time are different in different reference frames or in a curved space, here, the effect even happens in one reference frame and/or in a flat space. This quantum effect that a distant clock must be uncertain provides a new explanation to the dark energy. 


\section{Dynamical system under physical clock}

To study the impact of the physical clock to a dynamical universe system evolving with it, we consider that a whole system is defined by including a clock field $S_{T}[T(x)]$ and the rest of the (to-be-measured) universe $S_{U}[\varphi(x)]$ sharing the external parameter $x$. These two systems are assumed independent and do not interact with each other, while the time evolution of the rest of the universe $S_{U}$ is with respect to the clock field. So the action of the whole system is separable [10-12]

$$
S=S_{U}+S_{T}
$$

Before studying the system, let us first briefly proof that the system $S$ is semi-classically equivalent to the to-be-measured system $S_{U}$ where the conventional parameter time is used. Without loss of generality, considering the to-be-measured system is a (one parameter) mechanical system $S_{U}[\varphi(\tau)]=\int d \tau \frac{1}{2}\left(\partial_{\tau} \varphi\right)^{2}-V[\varphi]$, and the physical clock is $S_{T}=\int d \tau \frac{1}{2}\left(\partial_{\tau} T\right)^{2}$, then the partition function of the whole system is

$$
Z=\int \mathcal{D} \varphi \mathcal{D} T e^{-\left(S_{U}+S_{T}\right)}
$$

The functional integral $\int \mathcal{D} T$ of physical clock can be calculated by the mean field approximation,

$$
Z \stackrel{M F}{\approx} \int \mathcal{D} \varphi e^{-S_{\mathrm{eff}}}
$$

Up to an unimportant constant, the effective action could be written as

$$
S_{\text {eff }}\left[\varphi, \frac{\delta \varphi}{\delta T}\right]=\int d T \frac{1}{2} \mathcal{M}\left(\frac{\delta \varphi}{\delta T}\right)^{2}-V[\varphi]+\text { constant }
$$

in which $\mathcal{M}=\left\langle\left\|\frac{\partial \tau}{\partial T}\right\|\left(\frac{\partial T}{\partial \tau}\right)^{2}\right\rangle_{M F}$ is a constant depending on the integration constant of the mean field value of $T(\tau)$. It is easy to see that the mean field value of $T(\tau)$ is a monotonically increasing function of $\tau$, in this sense, the quantum clock becomes classical. The effective action now reproduces the classical structure of action $S_{U}$, only formally, the functional derivative with respect to the clock time $T(\tau)$ replaces the conventional derivative with respect to the parameter time $\tau$.

The one-parameter proof can be generalized to a multi-parameter case, in which not only time but also spatial coordinates are measured by physical instruments. The multiparameter case that puts the time and space on an equal footing is equivalent to generalize the idea of quantum clock to a quantum reference frame [13].

Generally speaking, the system $S=S_{U}+S_{T}$ corresponds to a system satisfying a timeless Wheeler-DeWitt equation, while the system $S_{\text {eff }}$ corresponds to an emergent effective system (from $S$ ) satisfying the Schrödinger equation in which external parameter time is used. It is worth stressing that the theory $S$ and $S_{U}$ are equivalent at semi-classical level, but they are different at quantum level. The rest of the paper is based on the system $S=S_{U}+S_{T}$. 


\subsection{Zero-point energy}

Since the notion of time now is changed, the notion of energy changes accordingly. Energy is defined as a conserved quantity under the time shift, and hence formally, the conventional derivative in the energy definition $E \sim \frac{\partial}{\partial t}$ is replaced by a functional derivative $E \sim \frac{\delta}{\delta T}$. Note that the action $S_{T}$ is quadratic in $T$, and $S_{U}$ does not explicitly contain $T$, so the vacuum energy of the system is

$$
\langle E\rangle=-\frac{\delta \ln Z}{\delta T} \approx \frac{\delta S}{\delta T}=0 .
$$

This result means that the zero-point vacuum energy of the whole system $S$ is vanished under the physical time $T$, which explains the first part of the problem. The physical reason for that the zero-point energy $\frac{1}{2} \sum_{k} \hbar \omega_{k}$ does not appear is transparent, because here time is the internal field $T(x)$ undergoing quantum fluctuation but an external parameter time $x_{0}$, the zero-point energy can not be seen when the observer is holding a physical clock that is also quantum fluctuating.

\subsection{Vacuum energy fluctuations}

That is not to say the vacuum is trivial, according to the uncertainty principle, an apparent energy variance emerges out of the void related to the intrinsic time uncertainty eq. (2.5), i.e. $\left\langle\delta E^{2}\right\rangle=\left\langle E^{2}\right\rangle-\langle E\rangle^{2}=\left\langle E^{2}\right\rangle=\frac{\delta^{2} S}{\delta T^{2}} \neq 0$. The further the distance, the more uncertain the time, and the larger the energy variance out of the void. The vacuum energy fluctuation in a 4 -volume element can be given by

$$
\begin{aligned}
\langle\delta E(x) \delta E(0)\rangle d^{4} x & =-\frac{\delta^{2} \ln Z}{\delta T(x) \delta T(0)} d^{4} x \\
& \approx \frac{\delta^{2} S}{\delta T(x) \delta T(0)} d^{4} x=\partial_{x}^{2} \delta^{4}(x) d^{4} x .
\end{aligned}
$$

At tree level approximation, we have approximately used $\ln Z \approx-S$, so the leading result is expressed in terms of a widthless Dirac delta function, while it actually has a non-zero width. This calculation can be performed by first rewrite the Dirac delta distribution as a limit of the Gaussian distribution, i.e. $\delta(x)=\lim _{a \rightarrow 0} \frac{1}{a \sqrt{\pi}} e^{-\frac{x^{2}}{a^{2}}}$, doing the derivatives and finally taking the zero width limit of the Gaussian distribution back to the Dirac delta distribution,

$$
\begin{aligned}
\langle\delta E(x) \delta E(0)\rangle d^{4} x & =\lim _{a \rightarrow 0} \partial_{x}^{2}\left(\frac{1}{a \sqrt{\pi}} e^{-\frac{x^{2}}{a^{2}}}\right)^{4} d^{4} x \\
& =64 a^{-4}|x-0|^{2} \delta^{4}(x) d^{4} x
\end{aligned}
$$

The width of the Gaussian distribution $a$ is an ultraviolet cut-off, the most natural choice is the Planck length $a=L_{P}$. If the distance $|x-0|$ is large, the energy fluctuation becomes considerable when it is at cosmic scale.

To regulate the result, an infrared cut-off is required, a natural choice is the Hubble length $|x-0|=L_{H}$, as the largest distance we could see, i.e. cosmic horizon. Therefore, 
when we fix the radius $|x-0|=L_{H}$ and integrate over $x$, then the total energy fluctuation of the vacuum in the Hubble scale volume is obtained

$$
\left\langle\delta E^{2}\right\rangle=64 \int d^{4} x L_{P}^{-4} L_{H}^{2} \delta^{4}(x)=64 L_{P}^{-4} L_{H}^{2} .
$$

Then an averaged vacuum energy density (averaged in the 3-ball with fixed radius $|x-0|=$ $\left.L_{H}\right)$ due to the total vacuum energy fluctuation is predicted as

$$
\rho_{d e}=\frac{\sqrt{\left\langle\delta E^{2}\right\rangle}}{\frac{4 \pi}{3} L_{H}^{3}}=\frac{6}{\pi} L_{P}^{-2} L_{H}^{-2}
$$

and

$$
\Omega_{d e}=\frac{\rho_{d e}}{\rho_{c}}=\frac{2}{\pi} \approx 0.64,
$$

where $\rho_{c}=\frac{3 H^{2}}{8 \pi G}$ is the critical density, $H=L_{H}^{-1}$ is the Hubble's constant, $8 \pi G=L_{P}^{2}$ is the Newton's gravitational constant, and $\Omega_{d e}$ is the fraction of the effective vacuum energy. The leading order predicted $\Omega_{d e}$ is a little lower than the current best fit from the data of Planck satellite [14], but still within the allowed range. This result explains the second part of the problem.

There are several important remarks of this result to emphasize. (i) We have considered the question: what a vacuum energy fluctuation is seen by a distant observer in a homogeneous, isotropic and empty flat space, when the time is defined by a physical clock field $T(x)$. (ii) The coordinates $x$ in the action eq. (2.1) are just external parameters which can only be seen by an external classical observer outside the universe. The reason we only pick up "time" treating quantum mechanically and the spacetime coordinates treating as the external parameter is for simplicity, a more rigor and general quantum mechanical treatment is to put the space and time on an equal footing (quantum reference frame [13]), which does not dramatically change the result when we only focus on energy and time. (iii) The value of the results eq. (3.9) and (3.10) gives correct order, in fact, they indeed depend on the precise nature of the cut-offs, and at present, the numerical factors of the cut-offs are chosen as the most natural ones.

\subsection{The coincidence problem}

Since the action $S_{T}$ is quadratic in $T$, the higher order $(>2)$ functional derivative with respect to clock time $T$ are all vanished, i.e. $\frac{\delta\left\langle\delta E^{2}\right\rangle}{\delta T}=\frac{\delta^{3} S}{\delta T^{3}}=0$. As a result, the vacuum energy fluctuation does not evolve with the clock time, thus leading to the fraction $\Omega_{d e}$ does not vary with this clock time. It is a constant and is "always" comparable with the critical density. And as a vacuum energy, it is uniform and a constant, moreover, its equation of state strictly equal to -1 and does not vary with time either.

Note that, because in our framework the time is a local internal observable, so those old notions of evolution in the standard cosmology must be carefully reconsidered. The exact meaning of the time evolution of any quantities is that their functional derivative with respect to the clock time $T$ is non-vanished. However, for example, the Hubble parameter $H(t)$, the fraction $\Omega_{i}(t)$ and the equation of state $w(t)$ as functions of parameter 
time only seen by an external classical observer have no physical meaning in our setting. So the infrared cut-off, the Hubble constant $H$ and/or Hubble length $L_{H}$ in eq. (3.9), in this sense, is really a constant.

As a consequence, we have had two statements: (i) in the standard cosmology, the matter density evolves with time, while the dark energy remains a constant; (ii) in this theory without coincidence problem, the matter density is "always" comparable with the dark energy density. It seems that they contradict each other, how could these two statements are both true, please do not immediately make an arbitrary judgment that it must be wrong. The key is again the notion of time, and the expansion of the universe is relative but absolute.

The view of a local internal observer is very different from that of an absolute external observer who feels a gradually diluting matter component under the cosmic expansion. If the universe is spatially flat $\Omega_{K}=0$, the matter density is always approximately $\Omega_{M} \approx 1-\Omega_{d e}$ seen by an internal observer at any epoch. In contrary to what one may think, here the matter density does not change under the local internal clock. Considering the universe is divided into two parts, one is a finite regime $\mathrm{A}$ in which an observer lives, and the regime $\mathrm{B}$ is the rest of the universe. The notion "now" in principle is a limit of regime A shrinking to infinitely small, but in practice the regime can be considered finite, i.e. the notion "near now" or "a near epoch" used above. The change in the regime B is defined relative to the clock in regime A which is external. While the change in the regime $\mathrm{A}$ is relative to the clock also in regime $\mathrm{A}$ which is internal. The consequence is that the internal observer does not see expansion of regime A with respect to the internal clock. Because the internal observer always lives in the regime A ("near now" regime), although he/she as an external observer can see changes in regime B, there seems an almost static matter density in the regime A, since his/her rulers and clocks are expanding correspondingly, that is the reason the internal observer always see the matter density does not vary with time and always comparable with the apparent "dark energy". In this sense, the Hubble volume is seen unchanged with local internal time, and hence the Hubble length as the infrared cutoff always remains $L_{P}=H^{-1}$. In the standard external observer's interpretation, it is a problem of coincidence, but in a local internal observer's view, the densities do not vary with their clocks, and the coincident redshift $z_{c}$ is always relatively small.

It is worth emphasizing that "always comparable" does not mean these two as real components of the universe would be scaled in the same way under expansion seen by an external observer, since it is impossible to be consistent with many observations such as the galaxies formation and the growth of large scale structure. In certain sense, the evolution of the observable universe gives place to the evolution/scaling with redshift. The cosmic acceleration in fact is an apparent quantum cosmic variance, and we are not living in a special epoch, whenever an (internal) observation is performed, the mirage "dark energy" is always seen being of the order of the matter density. What the internal observer sees is very different from that of the standard external observer. In this sense, the resolution of the coincidence problem is not dynamical, the key is again the notion of time. 


\section{Distance-redshift relation}

The notion of time is a key to the dark energy problem, this can be seen also from analyzing what we really measure in those dark energy observations [9]. Up to date, the measurement indications for the existence of dark energy (e.g. the supernovae Ia and Cosmic Microwave Background (CMB) ) come from the distance measurements $D$ and their relation to the redshift $z$. In fact, we have not measured the dark energy and its equation of state directly. The two observables $(z, D)$ are independently measured. The distance of supernovae is determined by observing the "luminosity distance", and the CMB is again by the "angular diameter distance" measurement on the last scattering surface. The redshift of the supernovae and CMB relate to the frequencies or time measurement of distant objects. Most of the data satisfying the Hubble's law, which states a linear dependence between the distance and redshift, is at low redshift regime. It is the high redshift observations that detect a distance which is significantly larger than the expected value in a flat matter dominanted or curvature dominated universe with the same Hubble constant. The unusual $D(z)$ relation at high redshift then infers the existence of dark energy by assuming the validity of general relativity. Until now there is no other test to tell us whether the dark energy is truly a new component of universe or simply a misunderstanding of the distant measurements, especially at high redshift (far off distance) regime, for example the cosmic scale distant frequency or time measurement. In fact, there is no experimental basis to state that a distant measured frequency or distant clock is exactly the same as the native ones.

Let us assume the time uncertainty previously considered in flat space is still (at least approximately) correct in the Hubble's expanding universe. At small redshift $z \equiv a_{0} / a-1$, the distance-redshift relation $D(z)$ is given by

$$
H_{0} D=z+\frac{1}{2} z^{2}+\ldots
$$

where $H_{0}$ is the Hubble's constant at $z=0, D$ is the luminosity distance. Since the distant frequency or redshift measurement has been reconsidered, such effect will give a modification to the distance-redshift relation. The distant time uncertainty does not change the central value of the spectral line or redshift, only broadens it and gives a non-vanishing variance $\left\langle\delta z^{2}\right\rangle \neq 0$. As a consequence, the distance-redshift relation $D(z)$ is modified at the order $\mathcal{O}\left(z^{2}\right)$ by an extra positive contribution

$$
H_{0} D=\langle z\rangle+\frac{1}{2}\left(\langle z\rangle^{2}+\left\langle\delta z^{2}\right\rangle\right)+\ldots
$$

in which we have used $\left\langle z^{2}\right\rangle=\langle z\rangle^{2}+\left\langle\delta z^{2}\right\rangle$. It is the extra positive contribution coming from the distant time/simultaneity uncertainty makes the effective "dark energy" behave repulsive.

Now we calculate the redshift variance $\left\langle\delta z^{2}\right\rangle$ from the uncertainty of distant clock given by eq. (2.4). If we work in Minkovski space, i.e. $\left(i x_{0}, x_{1}, x_{2}, x_{3}\right)$, the path integral eq. (2.3) becomes complex, when the distance $|x-y|$ (and corresponding $V_{\mathbb{R}_{3}} \sim|x-y|_{\text {max }}^{3}$ ) tends 
to infinity, we have a limit

$$
\lim _{|x-y| \rightarrow \infty} \sqrt{\frac{V_{\mathbb{R}_{3}}}{2 \pi|x-y|}} e^{\frac{i V_{\mathbb{R}_{3}}[T(x)-T(y)]^{2}}{2|x-y|}}=\lim _{|x-y| \rightarrow \infty} \frac{1}{\sqrt{2 \pi}(\delta T)} e^{\frac{i[T(x)-T(y)]^{2}}{2(\delta T)^{2}}}=e^{i \frac{\pi}{4}} \delta[T(x)-T(y)],
$$

where $\delta[T(x)-T(y)]$ is the Dirac delta function. Note that the phase factor of the formula tends to a constant $\pi / 4$ in this limit, and hence the redshift variance over redshift squared tends to $2 / \pi$,

$$
\lim _{|x-y| \rightarrow \infty} \frac{(\delta T)^{2}}{[T(x)-T(y)]^{2}}=\lim _{z \rightarrow \infty} \frac{\left\langle\delta z^{2}\right\rangle}{\langle z\rangle^{2}}=\frac{2}{\pi},
$$

in which we have used $\left\langle\delta z^{2}\right\rangle=\frac{\left\langle\delta T^{2}\right\rangle}{\langle T(x)\rangle^{2}}$ and $\langle z\rangle=\frac{\langle T(y)-T(x)\rangle}{\langle T(x)\rangle}$.

The linear relationship between the distant spectral line width and redshift (at high redshift) is an important prediction of the idea which can be tested by observations. Then we have a modified distance-redshift relation, the eq. (4.2) becomes

$$
H_{0} D=\langle z\rangle+\frac{1}{2}\left(1-q_{0}\right)\langle z\rangle^{2}+\ldots=\langle z\rangle+\frac{1}{2}\left(1+\frac{2}{\pi}\right)\langle z\rangle^{2}+\ldots
$$

Therefore, in a flat universe without ordinary matter (pressureless matter and radiation), the uncertainty of distant clock induces a redshift independent deceleration parameter $q_{0}=-\frac{2}{\pi}<0$, which makes the flat empty universe seem accelerating and being dominated by "dark energy" with fraction $\Omega_{d e}=\frac{2}{\pi}$. This result deduced from an independent calculation agrees with eq. (3.10).

In the flat universe $\Omega_{K}=0$, if the contribution of ordinary pressureless matter is taken into account, which evolves as $\Omega_{M}(1+z)^{3}$ with the redshift $z$, then we have

$$
H_{0} D=\langle z\rangle+\frac{1}{2}\left(1-\Omega_{M}(1+z)^{3}+\Omega_{d e}\right)\langle z\rangle^{2}+\ldots
$$

So the universe is always seen become accelerating at a relatively small redshift, at $-q_{0}=$ $-\Omega_{M}(1+z)^{3}+\Omega_{d e}=0$, i.e. $z_{c} \approx 0.3$ with respect to current epoch.

\section{Conclusions}

Finally, let us summarize the paper. In this paper, we retain the equivalence principle and abandon the standard interpretation of parameter time in quantum mechanics. The quantum spatial evolution makes the physical clock field fuzzy as the distance increases, leading to a quantum uncertainty of distant clock time. The idea of reinterpretation of time solves the dark energy problem. This theory tells us that the observed dark energy is a quantum effect connected to the quantum uncertainty of spatially separated clocks. The apparent vacuum energy fluctuation is inevitable if we use the physical clock redefining the time, and the result fits the observation well. This framework requires a modification of the standard quantum mechanics. Although the global parameters of quantum mechanics are necessary by its intrinsic structure, there is no prior reason to interpret them as time, time here is what we read from a physical clock that needs to be described quantum mechanically. The modified quantum framework requires a relational interpretation in terms of entangled state which is more natural than its standard absolute interpretation, 
since not only the to-be-measured system but also the measuring instruments such as the clock are both needed to be treated by quantum mechanics. In this sense, the WheelerDeWitt equation plays a more fundamental role than the emerged Schrödinger equation. And most importantly, this idea provides a touchstone to the longstanding difficulty of reconciliation of the inconsistency between general relativity and quantum mechanics.

\section{Acknowledgments}

This work was supported in part by the National Science Foundation of China (NSFC) under Grant No.11205149.

Open Access. This article is distributed under the terms of the Creative Commons Attribution License (CC-BY 4.0), which permits any use, distribution and reproduction in any medium, provided the original author(s) and source are credited.

\section{References}

[1] Supernova Search Team collaboration, A.G. Riess et al., Observational evidence from supernovae for an accelerating universe and a cosmological constant, Astron. J. 116 (1998) 1009 [astro-ph/9805201] [INSPIRE].

[2] Supernova Cosmology Project collaboration, S. Perlmutter et al., Measurements of $\Omega$ and $\Lambda$ from 42 high redshift supernovae, Astrophys. J. 517 (1999) 565 [astro-ph/9812133] [INSPIRE].

[3] P.J.E. Peebles and B. Ratra, The cosmological constant and dark energy, Rev. Mod. Phys. 75 (2003) 559 [astro-ph/0207347] [INSPIRE].

[4] S. Weinberg, The cosmological constant problem, Rev. Mod. Phys. 61 (1989) 1 [INSPIRE].

[5] E.J. Copeland, M. Sami and S. Tsujikawa, Dynamics of dark energy, Int. J. Mod. Phys. D 15 (2006) 1753 [hep-th/0603057] [INSPIRE].

[6] J. Polchinski, The cosmological constant and the string landscape, hep-th/0603249 [INSPIRE].

[7] R. Bousso, TASI lectures on the cosmological constant, Gen. Rel. Grav. 40 (2008) 607 [arXiv: 0708.4231] [INSPIRE].

[8] C. Rovelli, Quantum gravity, Cambridge University Press, Cambridge U.K. (2004) [InSPIRE].

[9] R. Durrer, What do we really know about dark energy?, Phil. Trans. Roy. Soc. Lond. A 369 (2011) 5102 [arXiv:1103.5331] [InSPIRE].

[10] C. Rovelli, Quantum mechanics without time: a model, Phys. Rev. D 42 (1990) 2638 [INSPIRE].

[11] D.N. Page and W.K. Wootters, Evolution without evolution: dynamics described by stationary observables, Phys. Rev. D 27 (1983) 2885 [InSPIRE].

[12] E. Moreva et al., Time from quantum entanglement: an experimental illustration, Phys. Rev. A 89 (2014) 052122 [arXiv:1310.4691] [INSPIRE].

[13] M.J. Luo, The cosmological constant problem and re-interpretation of time, Nucl. Phys. B 884 (2014) 344 [arXiv:1312.2759] [INSPIRE].

[14] Planck collaboration, P.A.R. Ade et al., Planck 2013 results. I. Overview of products and scientific results, Astron. Astrophys. 571 (2014) A1 [arXiv:1303.5062] [INSPIRE]. 